\title{
The Evolution of the Chern-Simons Vortices
}

\author{
Li-Bin $\mathrm{Fu}^{1, *}$, Yishi Duan ${ }^{2}$ and Hong Zhang ${ }^{2}$ \\ ${ }^{1}$ LCP, Institute of Applied Physics and Computational Mathematics, \\ P.O. Box 8009(26), Beijing 100088, P.R. China \\ 2 Physics Department, Lanzhou University, Lanzhou 730000, P.R. China
}

\begin{abstract}
Abstract

Based on the gauge potential decomposition theory and the $\phi$-mapping theory, the topological inner structure of the Chern-Simons-Higgs vortex has been showed in detail. The evolution of CSH vortices is studied from the topological properties of the Higgs scalar field. The vortices are found generating or annihilating at the limit points and encountering, splitting or merging at the bifurcation points of the scalar field $\phi$.
\end{abstract}

PACS number(s): 47.32.Cc, 11.15.-q, 02.40.PC

Keywords: vortices, evolution

*Email: fu_libin@mail.iapcm.ac.cn 


\section{INTRODUCTION}

In resent years, a great deal of work on the Abelian Chern-Simons-Higgs model in $2+1$ dimension has been done by many physicists 낸. This model has been widely used in many fields in physics, such as the fractional spin in quantum field theory [3,5], and the quantum Hall effect in condensed matter physics [6, []. Though it has been common to include the topological properties of the Abelian CSH vortex, the topological structure of this vortex has not been studied strictly. In this paper, based on the decomposition theory of gauge potential and the $\phi$-mapping theory, the inner structure of the CSH vortex and its evolution will be discussed in detail.

We know that the Abelian CSH Lagrangian density in $2+1$ dimensions is often expressed as

$$
L_{C S H}(\phi, A)=\frac{1}{4} \alpha \in^{\mu \nu \lambda} A_{\mu} F_{v \lambda}+\frac{1}{2} D \phi(D \phi)^{*}+V(\phi)
$$

where $\phi$ is the designated charged Higgs scalar field and $\frac{1}{4} \alpha \in^{\mu \nu \lambda} A_{\mu} F_{v \lambda}$ is so-called ChernSimons term. As have been pointed out by many physicists [8,9], the magnetic flux of the vortex is

$$
\Phi=\oint A_{i} d x^{i}=\int \frac{1}{2} \in^{i j} \partial_{i} A_{j} d x^{2}=\frac{2 \pi \hbar c}{e} n
$$

where $n$ is a topological index, characterizing the vortex configuration. $\Phi$ is also referred to as the topological charge.

In our point of view 10,11, the $U(1)$ gauge potential can be decomposed by the Higgs complex scalar field $\phi=\phi^{1}+i \phi^{2}$ as

$$
A_{\mu}=\frac{\hbar c}{e} \in^{a b} \partial_{\mu} n^{a} n^{b}+\partial_{\mu} \lambda
$$

in which $\lambda$ is only a phase factor, and $n$ is a unit vector field defined by

$$
n^{a}=\frac{\phi^{a}}{\|\phi\|}, \quad\|\phi\|=\left(\phi^{a} \phi^{a}\right)^{1 / 2}
$$

It can be proved that there exists a topological current 


$$
J^{\mu}=\frac{1}{2} \in^{\mu \nu \lambda} \partial_{\nu} A_{\lambda}=\frac{\hbar c}{2 e} \in^{\mu \nu \lambda} \in_{a b} \partial_{\mu} n^{a} \partial_{\nu} n^{b}
$$

Obviously, the current (1.4) is conserved. Following the $\phi$-mapping theory [10], it can be rigorously proved that

$$
J^{\mu}=\frac{2 \pi \hbar c}{e} \delta^{2}(\vec{\phi}) D^{\mu}\left(\frac{\phi}{x}\right)
$$

where the Jacobian $D^{\mu}\left(\frac{\phi}{x}\right)$ is defined as

$$
\epsilon^{a b} D^{\mu}\left(\frac{\phi}{x}\right)=\epsilon^{\mu \nu \lambda} \partial_{\nu} n^{a} \partial_{\lambda} n^{b}
$$

According to the $\delta$-function theory [12] and the $\phi$-mapping theory, one can prove that

$$
J^{\mu}=\left.\frac{2 \pi \hbar c}{e} \sum_{i=1}^{l} \beta_{i} \eta_{i} \delta^{2}\left(\vec{x}-\vec{z}_{i}\right) \frac{d x^{\mu}}{d t}\right|_{z_{i}}
$$

where $\vec{z}_{i}$ are the $i$-th regular zero points of $\vec{\phi}$, i.e. the solution of the following equation

$$
\phi^{a}(x)=0, \quad a=1,2,
$$

and the positive integer $\beta_{i}$ is the Hopf index 13 15 and $\eta_{i}=\operatorname{sign}\left(D(\vec{\phi} / \vec{x})_{z_{i}}\right)= \pm 1$ is Brouwer degree [16,17]. Then the density of topological charge can be wrote as

$$
\rho=J^{0}=\frac{2 \pi \hbar c}{e} \sum_{i=1}^{l} \beta_{i} \eta_{i} \delta^{2}\left(\vec{x}-\vec{z}_{i}\right) .
$$

Comparing the formula to (1.2) and considering Eq. (1.4), the total charge of the system given in (1.2) can be rewrote as

$$
Q=\int \rho(x) d^{2} x=\Phi_{0} \sum_{i=1}^{l} \beta_{i} \eta_{i}
$$

where $\Phi_{0}=\frac{2 \pi \hbar c}{e}$ is unit magnetic flux. And it is easy to see that the topological index $n$ in (1.2) has the following expression

$$
n=\sum_{i=1}^{l} \beta_{i} \eta_{i}
$$

It is obvious to see that the vortex configuration given in (1.2) is a multivortex solution which possess the inner structure described by expression (1.8). We can see vortex corresponds to $\eta_{i}=+1$, while anti-vortex corresponding to $\eta_{i}=-1$. 


\section{THE GENERATION AND ANNIHILATION OF VORTICES}

As being discussed before, the zeros of the vector field $\vec{\phi}$ play an important roles in describing the topological structure of the vortices. Now we begin investigating the properties of the zero points. As we knew before, if the Jacobian

$$
D^{0}\left(\frac{\phi}{x}\right) \neq 0
$$

we will have the isolated zeros of the vector field $\vec{\phi}$. However, when the condition fails, the above discussion will change in some way and lead to the branch process. We denote one of the zero points as $\left(t^{*}, \vec{z}_{i}\right)$. If the Jacobian

$$
\left.D^{1}\left(\frac{\phi}{x}\right)\right|_{\left(t^{*}, \vec{z}_{i}\right)} \neq 0
$$

we can use the Jacobian $D^{1}\left(\frac{\phi}{x}\right)$ instead of $D^{0}\left(\frac{\phi}{x}\right)$ for the purpose of using the implicit function theorem [18]. Then we have a unique solution of the equations (1.6) in the neighborhood of the limit point $\left(t^{*}, \vec{z}_{i}\right)$

$$
t=t\left(x^{1}\right), \quad x^{2}=x^{2}\left(x^{1}\right)
$$

with $t^{*}=t\left(z_{i}^{1}\right)$. We call the critical points $\left(t^{*}, \vec{z}_{i}\right)$ the limit points. In the present case, we know that

$$
\left.\frac{d x^{1}}{d t}\right|_{\left(t^{*}, \vec{z}_{i}\right)}=\left.\frac{D^{1}\left(\frac{\phi}{x}\right)}{D\left(\frac{\phi}{x}\right)}\right|_{\left(t^{*}, \vec{z}_{i}\right)}=\infty
$$

i.e.,

$$
\left.\frac{d t}{d x^{1}}\right|_{\left(t^{*}, \vec{z}_{i}\right)}=0
$$

Then, the Taylor expansion of $t=t\left(x^{1}\right)$ at the limit point $\left(t^{*}, \vec{z}_{i}\right)$ is 19

$$
t-t^{*}=\left.\frac{1}{2} \frac{d^{2} t}{\left(d x^{1}\right)^{2}}\right|_{\left(t^{*}, \vec{z}_{i}\right)}\left(x^{1}-x_{i}^{1}\right)^{2}
$$

which is a parabola in $x^{1}-t$ plane. From Eq.(2.14) we can obtain two solutions $x_{1}^{1}(t)$ and $x_{2}^{1}(t)$, which give two branch solutions (worldlines of vortices). If $\left.\frac{d^{2} t}{\left(d x^{1}\right)^{2}}\right|_{\left(t^{*}, \vec{z}_{i}\right)}>0$, we have 
the branch solutions for $t>t^{*}$ [see Fig. 1(a)]; otherwise, we have the branch solutions for $t<t^{*}$ [see Fig. 1(b)]. These two cases are related to the origin annihilate of the vortices.

One of the result of Eq. (2.12), that the velocity of vortices are infinite when they are annihilating, agrees with the fact obtained by Bray [20] who has a scaling argument associated with point defects final annihilation which leads to large velocity tail. From Eq. (2.12), we also get a new result that the velocity of vortices is infinite when they are generating, which is gained only from the topology of the scalar fields.

Since the topological current is identically conserved, the topological charge of these two generated or annihilated vortices must be opposite at the limit point, i.e.

$$
\beta_{i_{1}} \eta_{i_{1}}=-\beta_{i_{2}} \eta_{i_{2}}
$$

which shows that $\beta_{i_{1}}=\beta_{i_{2}}$ and $\eta_{i_{1}}=-\eta_{i_{2}}$. One can see that the fact the Brouwer degree $\eta$ is indefinite at the limit points implies that it can change discontinuously at limit points along the worldlines of vortices (from \pm 1 to $\mp 1$ ). It is easy to see from Fig. 1: when $x^{1}>z_{i}^{1}$, $\eta_{i_{1}}= \pm 1 ;$ when $x^{1}<z_{i}^{1}, \eta_{i_{2}}=\mp 1$.

For a limit point it is required that $\left.D^{1}\left(\frac{\phi}{x}\right)\right|_{\left(t^{*}, \vec{z}_{i}\right)} \neq 0$. As to a bifurcation point [21], it must satisfy a more complex condition. This case will be discussed in the following section.

\section{THE BIFURCATION OF VORTICES VELOCITY FIELD}

In this section we have the restrictions of Eqs. (1.6) at the bifurcation points $\left(t^{*}, \vec{z}_{i}\right)$,

$$
\left.D\left(\frac{\phi}{x}\right)\right|_{z_{i}}=0,\left.\quad D^{1}\left(\frac{\phi}{x}\right)\right|_{z_{i}}=0
$$

which leads to an important fact that the function relationship between $t$ and $x^{1}$ is not unique in the neighborhood of the bifurcation point $\left(t^{*}, \vec{z}_{i}\right)$. It is easy to see that

$$
V^{1}=\frac{d x^{1}}{d t}=\left.\frac{D^{1}\left(\frac{\phi}{x}\right)}{D\left(\frac{\phi}{x}\right)}\right|_{z_{i}}
$$

which under (3.16) directly shows that the direction of the integral curve of (3.17) is indefinite at $\left(t^{*}, \vec{z}_{i}\right)$, i.e., the velocity field of vortices is indefinite at the point $\left(t^{*}, \vec{z}_{i}\right)$. This is why the very point $\left(t^{*}, \vec{z}_{i}\right)$ is called a bifurcation point. 
Assume that the bifurcation point $\left(t^{*}, \vec{z}_{i}\right)$ has been found from Eqs. (1.6) and (3.16). We know that, at the bifurcation point $\left(t^{*}, \vec{z}_{i}\right)$, the rank of the Jacobian matrix $[\partial \phi / \partial x]$ is 1. In addition, according to the $\phi$-mapping theory, the Taylor expansion of the solution of Eq. (1.6) in the neighborhood of the bifurcation point $\left(t^{*}, \vec{z}_{i}\right)$ can be expressed as $[19$

$$
A\left(x^{1}-x_{i}^{1}\right)^{2}+2 B\left(x^{1}-x_{i}^{1}\right)\left(t-t^{*}\right)+C\left(t-t^{*}\right)^{2}=0
$$

which leads to

$$
A\left(\frac{d x^{1}}{d t}\right)^{2}+2 B \frac{d x^{1}}{d t}+C=0
$$

and

$$
C\left(\frac{d t}{d x^{1}}\right)^{2}+2 B \frac{d t}{d x^{1}}+A=0
$$

where $A, B$ and $C$ are three constants. The solutions of Eq. (3.19) or Eq. (3.20) give different directions of the branch curves (worldlines of vortices) at the bifurcation point. There are four possible cases, which will show the physical meanings of the bifurcation points.

Case $1(A \neq 0)$ : For $\Delta=4\left(B^{2}-A C\right)>0$ from Eq. (3.19) we get two different directions of the velocity field of vortices

$$
\left.\frac{d x^{1}}{d t}\right|_{1,2}=\frac{-B \pm \sqrt{B^{2}-A C}}{A} .
$$

which is shown in Fig. 2, where two worldlines of two vortices intersect with different directions at the bifurcation point. This shows that two vortices encounter and then depart at the bifurcation point.

Case $2(A \neq 0)$ : For $\Delta=4\left(B^{2}-A C\right)=0$ from Eq. (3.19) we get only one direction of the velocity field of vortices

$$
\left.\frac{d x^{1}}{d t}\right|_{1,2}=-\frac{B}{A}
$$

which includes three important cases. (a) Two worldlines tangentially contact, i.e. two vortices tangentially encounter at the bifurcation point (see Fig. 3(a)). (b) Two worldlines 
merge into one worldline, i.e. two vortices merge into one vortex at the bifurcation point (see Fig. 3(b)). (c) One worldline resolves into two worldlines, i.e. one vortex splits into two vortices at the bifurcation point. (see Fig. 3(c)).

Case $3(A=0, C \neq 0)$ : For $\Delta=4\left(B^{2}-A C\right)=0$ from Eq. (3.20) we have

$$
\left.\frac{d t}{d x^{1}}\right|_{1,2}=\frac{-B \pm \sqrt{B^{2}-A C}}{C}=0, \quad-\frac{2 B}{C} .
$$

There are two important cases: (a) One worldline resolves into three worldlines, i.e. one vortex splits into three vortices at the bifurcation point (see Fig. 4(a)). (b) Three worldlines merge into one worldline, i.e. three vortices merge into one vortex at the bifurcation point (see Fig. 4(b)).

Case $4(A=C=0)$ : Eq. (3.19) and Eq. (3.20) gives respectively

$$
\frac{d x^{1}}{d t}=0, \quad \frac{d t}{d x^{1}}=0 .
$$

This case is obvious as Fig. 5 and is similar to case 3 .

The above solutions reveal the evolution of the vortices. Besides the encountering of the vortices, i.e. two vortices encounter and then depart at the bifurcation point along different branch curves (see Fig. 2 and Fig. 3(a)), it also includes spliting and merging of vortices. When a multicharged vortex moves through the bifurcation point, it may split into several vortices along different branch curves (see Fig. 3(c), Fig. 4(a) and Fig. 5(b)). On the contrary, several vortices can merge into one vortex at the bifurcation point (see Fig. 3(b), Fig. 4(b)).

From Eqs. (3.23) and (3.24), we find that there are two branches includes the case that the velocity of vortices is infinite at the bifurcation point, i.e. when one vortex splits into three vortices or three merge into one, there must exists a pair of vortices whose velocity is infinite at the bifurcation point. It seems that these cases are associated with the process of generating or annihilating. This is pointed to be verified in future.

The identical conversation of the topological charge shows the sum of the topological charge of these final vortices must be equal to that of the original vortices at the bifurcation point, i.e. 


$$
\sum_{i} \beta_{l_{i}} \eta_{l_{i}}=\sum_{f} \beta_{l_{f}} \eta_{l_{f}}
$$

for fixed $l$. Furthermore, from above studies, we see that the generation, annihilation and bifurcation of vortices are not gradual change, but sudden change at the critical points.

\section{CONCLUSION}

Firstly, we obtain the inner topological structure of Chern-Simons vortex. The multicharged vortex has been found at the every zero point of the Higgs scalar field $\phi$ under the condition that the Jacobian determinate $D(\phi / x) \neq 0$. One also shows that the charge of the vortex is determined by Hopf indices and Brouwer degrees. Secondly, we conclude that there

exist crucial cases of branch processes in the evolution of the vortices when $D\left(\frac{\phi}{x}\right)=0$, i.e., $\eta_{i}$ is indefinite. This means that the vortices generate or annihilate at the limit points and encounter, split or merge at the bifurcation points of the Higgs scalar fields, which shows that the vortices system is unstable at these branch points. Here we must point out that there exist two restrictions of the evolution of vortices. One restriction is the conservation of the topological charge of the vortices during the branch process (see Eqs. (2.15) and (3.25)), the other restriction is the number of different directions of the worldlines of vortices is at most four at the bifurcation points (see Eqs. (3.19), (3.20) ). Perhaps, the former is known before, but the later is pointed out for the first time. 


\section{REFERENCES}

[1] R. Jackiw and Erick J. Weinberg, Phys. Rew. Lett. 64 2234(1990),

[2] Jooyoo Hong, Yoobai Kim, and Pong Youl Pac, Phys. Rew. Lett. 64 2230(1990)

[3] Ashim Kumar Roy, Int. Joural of Modem Physics A, 12, 2343(1997)

[4] S.K. Kim and H. Min, Physics Letters B, 281, 81(1992)

[5] J. Fröhlich, M. Leupp, P. A. Marchetti, Comm. Math. Phys. 121, 177-233(1989)

[6] M. C. Diamantini, P. Sodano and C. A. Trugenberger, Nucl. Phys. B, B474, 641(1996)

[7] C. Duval, P. A. Horvathy and L. Palla, Phys. Rev. D, 52, 4700(1995)

[8] R. Jackiw and E. J. Weinberg, Phys. Rev. Lett. 642234 (1990)

[9] Ashim Kumar Roy, Int. J. Mod. Phys. A12 2343 (1997)

[10] Y. S. Duan, G. H. Yang, and Y. Jiang, Gen. Rel. Grav. 29, 715 (1997)

[11] Y. S. Duan, In Proc.Symposium on Yang-Mills gauge theories (Beijing)(1984)

[12] J. A. Schouton, Tensor analysis for Physicists (Claredon, Oxford, 1951)

[13] V. Guillemin and A. Pollack, Differential Topology (Prentice Hall, Englewood Cliffs, NJ) (1974)

[14] J. W. Milnor, Topology From the Differential Viewpoint(The University Press of Virginia, Charlottesville) (1965)

[15] B. A. Dubrovin, et. al., Modern Geometry -Methods and Applications (Springer-Verlag, New york), Part II (1985)

[16] Y. S. Duan and M. L. Ge, Sci. Sinica 11, 1072 (1979); C. Gu, Phys. Rep. C80, 251(1981)

[17] Y. S. Duan and S. L. Zhang, Int. J. Engng. Sci. 28, 689 (1990); 29, 153 (1991); 29, 1593 (1991); 30, 153 (1992). 
[18] E. Goursat, A Course in Mathematical Analysis, Vol1(transl. E. R. Hedrick), (1904).

[19] Y.S. Duan, S. Li and G.H. Yang, Nucl. Phys. B 514, 705 (1998)

[20] A. J. Bray, Phys. Rev. E 55, 5297 (1997)

[21] M. Kubicek and M. Marek, Computational Methods in Bifurcation Theory and Dissipative Structures (Springer-Verlag, New York, 1983)

\section{FIGURE CAPTIONS}

Fig. 1. Projecting the worldlines of vortices onto $\left(x^{1}-t\right)$-plane. (a) The branch solutions

for Eq. (2.14) when $\left.\frac{d^{2} t}{\left(d x^{1}\right)^{2}}\right|_{\left(t^{*}, \vec{z}_{i}\right)}>0$, i.e. a pair of vortices with opposite charges generate at the limit point, i.e. the origin of vortices. (b) The branch solutions for Eq. (2.14) when $\left.\frac{d^{2} t}{\left(d x^{1}\right)^{2}}\right|_{\left(t^{*}, \vec{z}_{i}\right)}<0$, i.e. a pair of vortices with opposite charges annihilate at the limit point.

Fig. 2. Projecting the worldlines of vortices onto $\left(x^{1}-t\right)$-plane. Two worldlines intersect with different directions at the bifurcation point, i.e. two vortices encounter at the bifurcation point.

Fig. 3. (a) Two worldlines tangentially contact, i.e. two vortices tangentially encounter at the bifurcation point. (b) Two worldlines merge into one worldline, i.e. two vortices merge into one vortices at the bifurcation point. (c) One worldline resolves into two worldlines, i.e. one vortex splits into two vortices at the bifurcation point.

Fig. 4. Two important cases of Eq. (3.23). (a) One worldline resolves into three worldlines, i.e. one vortex splits into three vortices at the bifurcation point. (b) Three worldlines merge into one worldline, i.e. three vortices merge into one vortex at the bifurcation point.

Fig. 5. Two worldlines intersect normally at the bifurcation point. This case is similar to Fig. 4. (a) Three vortices merge into one vortex at the bifurcation point. (b) One vortex splits into three vortices at the bifurcation point. 


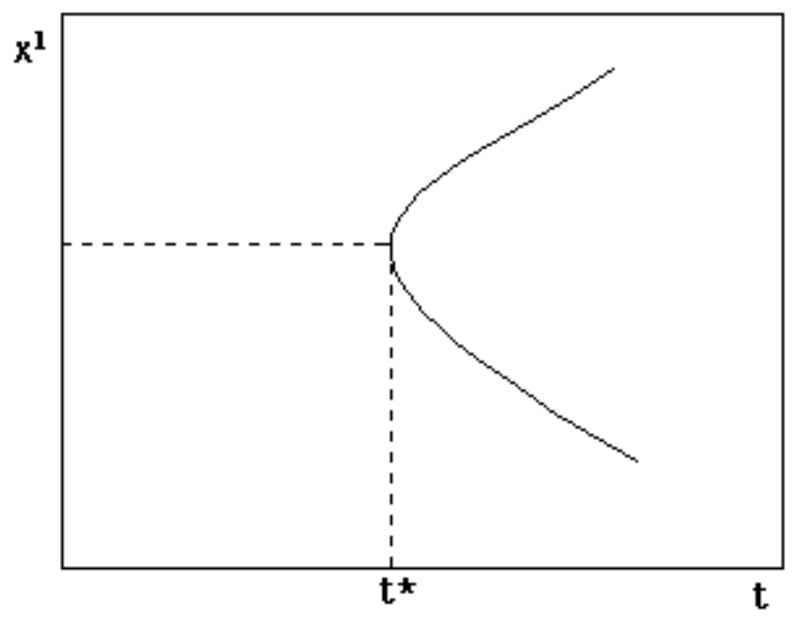

Fig. 1(a) 


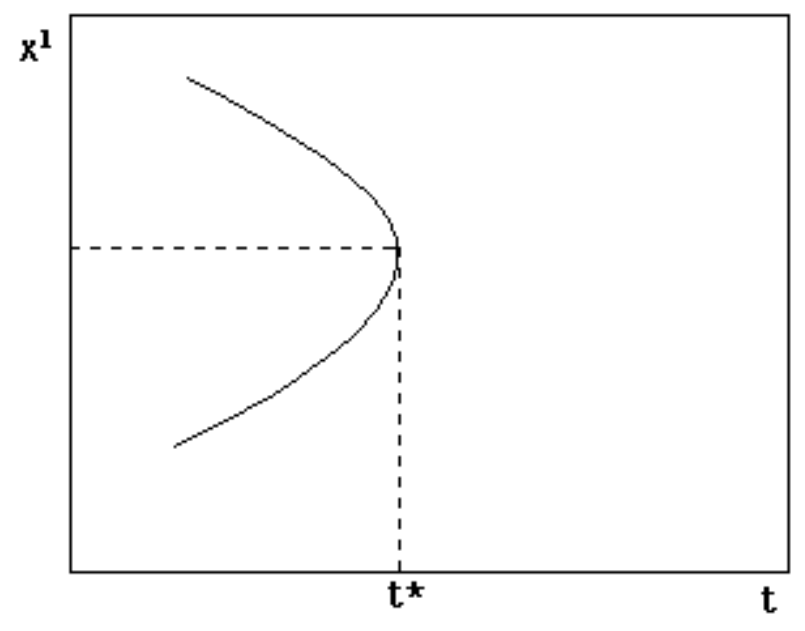

Fig. 1(b) 


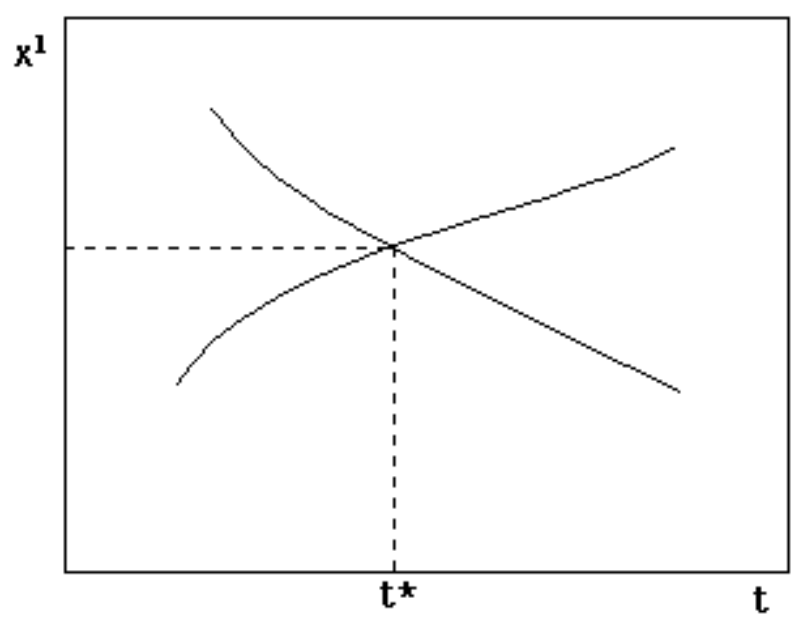

Fig. 2 


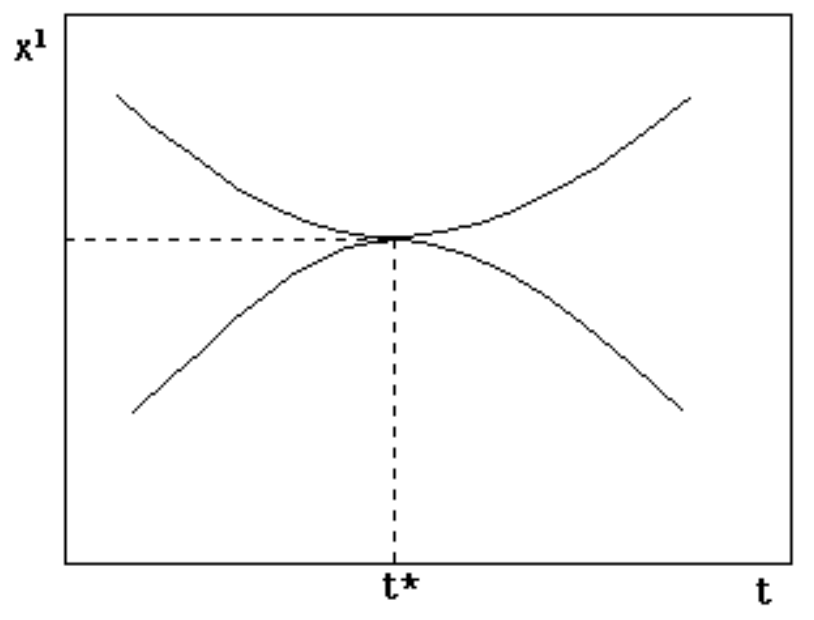

Fig. 3(a) 


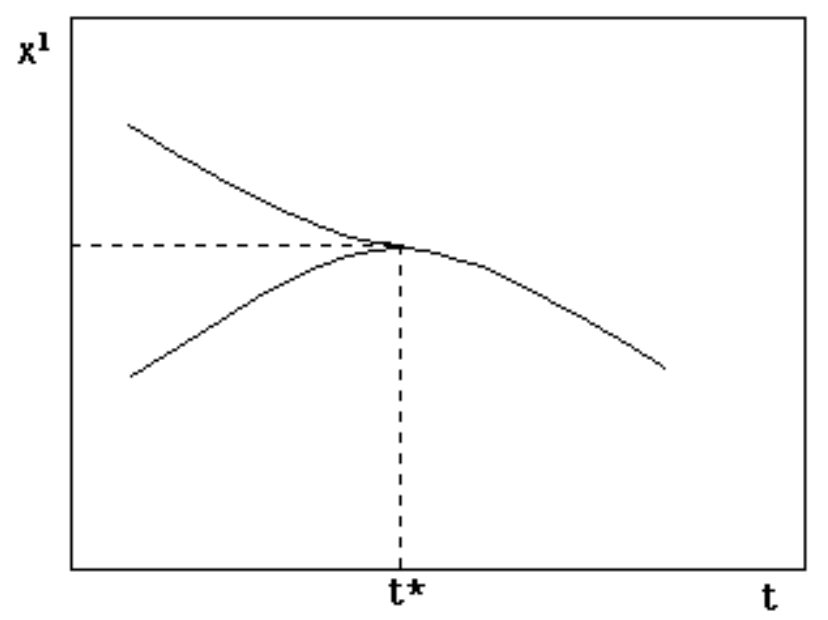

Fig. 3(b) 


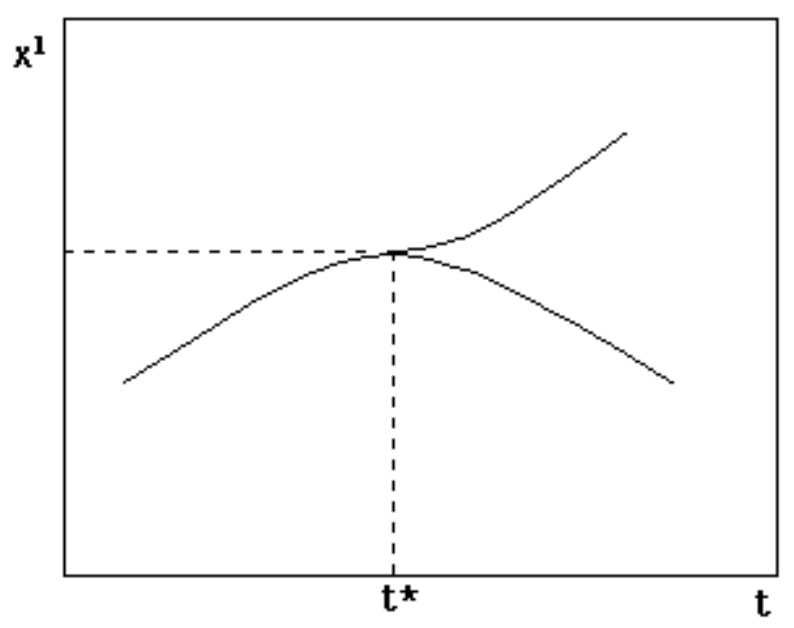

Fig. 3(c) 


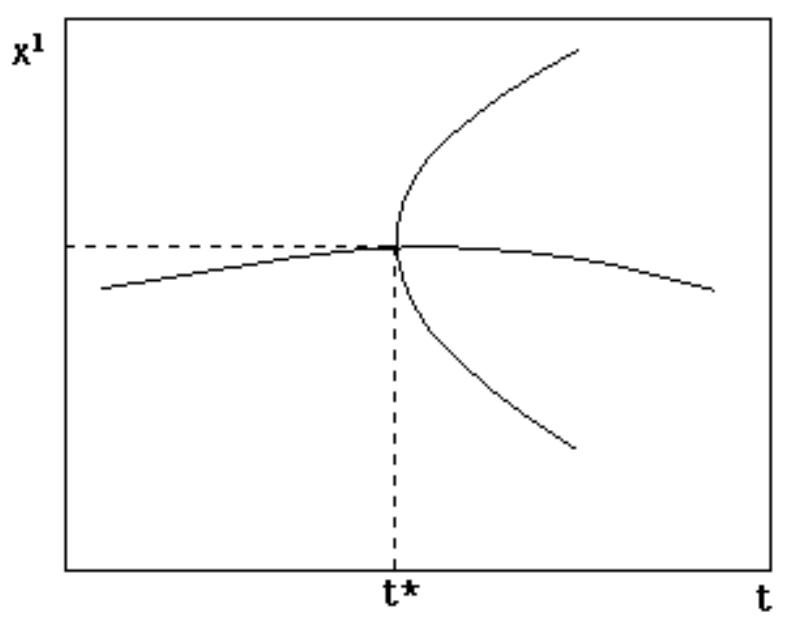

Fig. 4(a) 


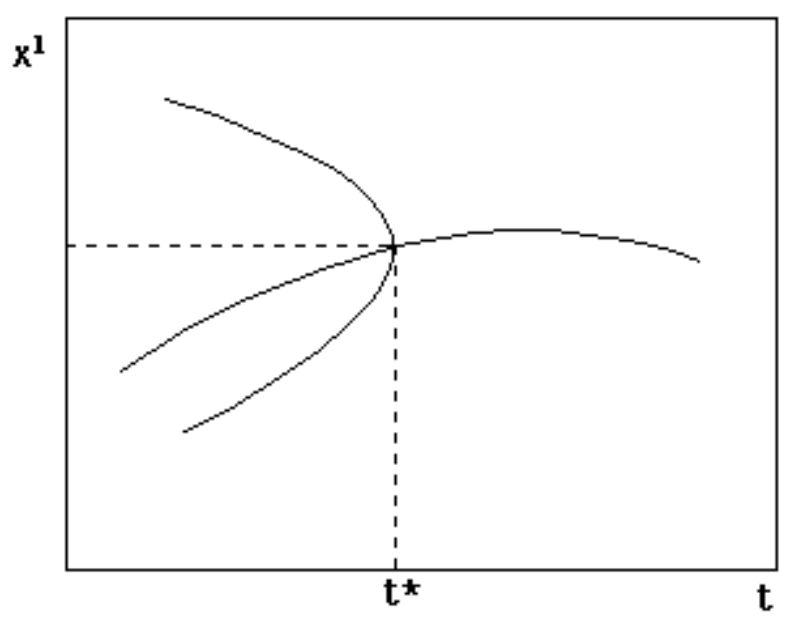

Fig. 4(b) 


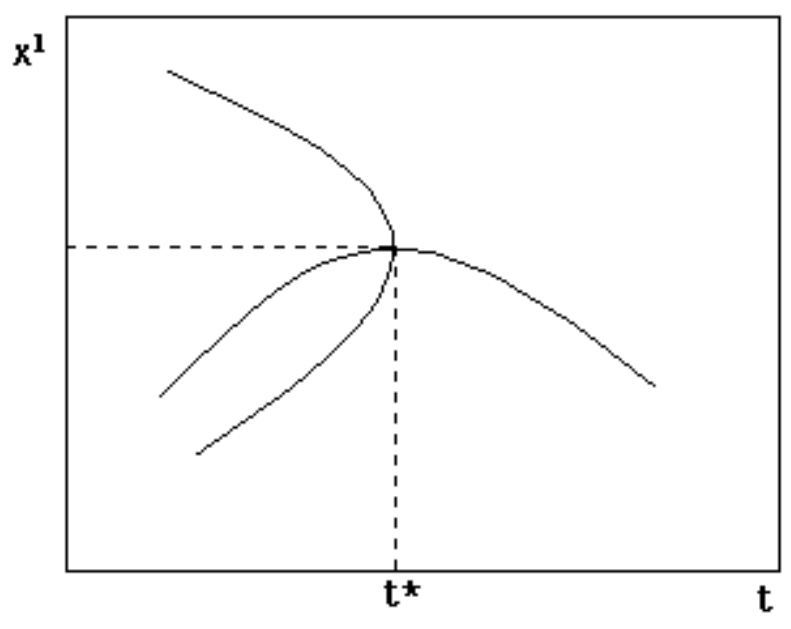

Fig. 5(a) 


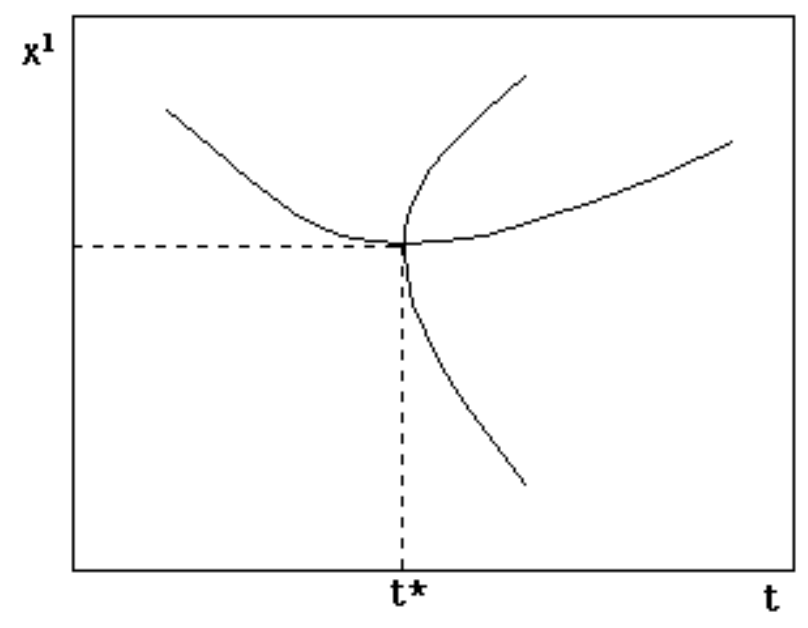

Fig. 5(b) 